\title{
9
}

\section{The case of Cabri-géomètre: learning geometry in a computer based environment}

\author{
Colette Laborde \\ Jean-Marie Laborde \\ Informatics and Applied Mathematics \\ Grenoble University, France
}

\section{ABSTRACT}

One of the reasons teachers may resist the use of computers in the mathematics classrooms is the change that this introduction implies about the kind of problems fostering the acquisition of knowledge by the pupil. Some old problems become uninteresting in the new environment. Conversely computers allow the design of some new problems that are impossible in a paper and pencil environment. In our paper we consider the computer as part of the "milieu didactique" : as such it offers specific representations of knowledge, specific facilities and specific feedback to the pupils. We will discuss the role of these characteristics on the way mathematical objects may be perceived on the interface by the pupils and on the solving processes of the pupils when they are confronted to problem situations on the computer, using the geometry program Cabri-géomètre.

Keywords: learner centred learning, drawing, graphics, innovation, microworld 


\section{INTRODUCTION}

Much research provides evidence that listening to the discourse of the teacher, to a clear presentation of mathematical content, does not guarantee the learning which was expected. According to constructivist theory which is widely used among mathematics educators, knowledge is actively built up by the cognizing subject when interacting with mathematical learning environments. This notion of environment must be taken in a broader sense that the environment is of material nature as well as of intellectual nature.

Problems are part of such environments, and may play an important role in the construction of mathematical knowledge by learners because they offer the opportunity of involving the learners' own ideas and of testing their efficiency and validity, when trying to solve the problem. Brousseau [1] considers the process of elaborating a solution by a learner as the interaction between the learner and a "milieu" which enables the learner to perform some given kinds of action in order to solve the problem and offer feedback to actions. In terms of systems, the "milieu" is the system working with, by "replying" to, the learner.

The graphical and computing possibilities of software now allow a reification or restatement of abstract objects and in particular of mathematical objects, as well as numerous possible operations and related feedback. The construction of knowledge may be fostered by problem solving in a computer based environment. But as Pea [2] claimed, the computer provides new tools for operating on these objects and therefore changes the objects themselves. Feedback can also be of a different nature; it may be very sophisticated in comparison with paper and pencil situation. All these points imply that designing a problem in a computer based environment requires a new analysis of mathematical objects, operations and feedback. This explains perhaps why teachers can be reluctant to use computers in their classrooms.

\section{THE NATURE OF GEOMETRY}

The nature of geometry is dual in that problems related to geometry may be of practical nature as well as theoretical. Although geometry was originally built as a way of controlling the relations with physical space. It has also been developed as a theoretical field dealing with abstract objects. 
One usual mediation of the theoretical objects of geometry is offered by graphical representations called "figures". The relation of the mathematicians with these representations is complex and in a sense reflects the dual nature of geometry; mathematicians drawn them, they act on them as if they were material objects (by means of a kind of experimentation), but their reasoning actually does not deal with them but with theoretical objects. This is why Parzysz [3] distinguishes the "figure" which is the theoretical referent (attached to a given geometrical theory : euclidean geometry, projective geometry...) from the "drawing" which is the material entity. But geometry software enabling the drawing of figures on the screen of computers forces us to refine this distinction in order to account for this new mediation.

\section{From drawing to figure}

As a material entity, a drawing is imperfect - the lines have a width, the straight lines are not really straight. But mathematics ignore these imperfections and work on a "idealised drawing". New questions are now becoming crucial for designers of graphical representations on the screen of a computer. For instance, to what extent are the imperfections of a drawing considered as noise by the users and do not prevent them from having access to the idealized drawing? To what extent is a sequence of small segments accepted as representing a straight line? The computer indeed reveals a phenomenon which is of importance for the pupils in a paper and pencil situation. For the latter the process of eliminating the imperfections of a real drawing is not so spontaneous as for mathematicians because it actually requires mathematical knowledge. Mathematicians know that a circle and a tangent line do not have in common a segment but only a point; they know that the tangent is perpendicular to the radius and they are able to infer this from the drawing even if the angle is not exactly right. But the move of a conceptual nature from the actual drawing to an idealized drawing is not spontaneous for pupils.

An idealized drawing may give rise to several figures depending on the features which are relevant for the problem to be solved. The referent attached to a drawing cannot be inferred only from the drawing but must be given by a text in a discursive way. There are two reasons for this. Firstly, some properties of the drawing may be irrelevant. For instance some relations which are apparent on the drawing may not be part of the figure, for instance the size of the sides a triangle may be not relevant for the problem to be solved, and the position of the drawing in the sheet of paper is usually without any link with the geometrical problem. Only 
some features of the drawing are relevant for the problem to be solved. And secondly, an important feature of geometrical figures is that they involve elements varying in subsets of the plane. A drawing itself cannot account for the variability of its elements.

\section{The geometrical figure behind the screen}

Computers have been used to design programs making apparent the multiplicity of drawings attached to a given geometrical figure. This has been done by several means like programming language or repeat facilities. Improved interfaces now allow direct manipulation while all geometrical properties used to construct the drawing are preserved.

A common feature of these programs is their use of an explicit description of the figures : a drawing produced on the screen is the result of a process performed by the user who makes explicit the definition of the referent. Such programs differ from drawing tools like MacPaint in which the process of construction of the drawing involves only action on the physical screen and does not require a description of the referent, that is of relations between elements. Nevertheless because the design of a geometry software presents some specificities, the referent of a drawing itself cannot be completely identified with the usual referent in Euclidean geometry. In software geometry, the figure is determined by a construction process made of primitives and by the operations which are possible to perform.

A new kind of referent is thus created by such geometry programs, but also new problems may arise because of the novelty of the objects and operations that are possible on these objects. But referents and problems actually differ from one software to another one according to the software design. This question becomes clearer when discussed through the example of Cabri-géomètre.

\section{The Cabri-figure and the Cabri-drawing}

Because the drawing made with Cabri-géomètre on the screen has specific features, it will be denoted by the term Cabri-drawing and the object of the theory to which it refers by Cabri-figure. Two kind of primitives are available to make a drawing in Cabri-géomètre:

- primitives of pure drawing which enable the user

a) to mark a point anywhere on the screen, just at the location shown by means of the cursor (which takes the form of a pen), we call this kind of point a basis point; 
b) to draw a straight line while pointing on the screen the position of two points but these points are not created as geometrical objects, we call this straight line a basis line; and

c) to draw a circular line called a basis circle;

- primitives based on geometrical properties which enable the user to draw objects not on a perceptive basis but on a geometrical basis : for example, the user can draw a perpendicular bisector of a segment in selecting the item perpendicular bisector in the menu Construction and in showing with the cursor the given segment.

When a Cabri-drawing is dragged, every constituent of the drawing which does not depend geometrically on the other ones does not move. For example, if a segment $\mathrm{AB}$ is drawn and if the user decides to mark its mid-point I only in showing a point correctly located from a visual point of view by means of the primitive basis point, this point I does not move when A or B is dragged (see figs. $1 \& 2$ ).

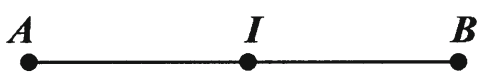

Figure 1: I is a basis point visually posed on $A B$

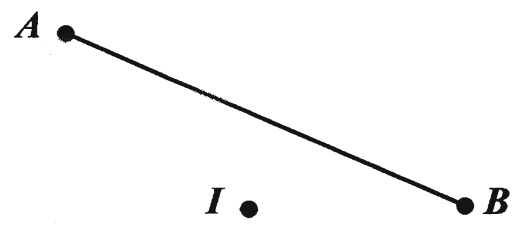

Figure 2: A is dragged, I does not follow $\mathrm{AB}$

In contrast if the mid-point is drawn by using the menu item mid-point its property of being the mid-point of $A B$ is preserved by the drag mode.

The combination of these two kinds of primitives and of the drag mode gives meaning to the notion of figure versus the notion of drawing. A drawing is not resistant to the dragging while a geometrical figure is the set of geometrical properties and the relations preserved by the drag mode. 7 But the theoretical object to which the Cabri-drawing refers presents some features which differ from those attached to the usual theoretical referent. The Cabri-figure is a result of a sequential description process, which introduces an order among the elements of the figure. So in a Cabri-figure some elements are basis elements or free elements on which the other elements are constructed. These free elements can be grasped by the mouse and dragged, they have a degree of freedom equal to 2 whereas a constructed element like the mid-point of a segment is completely dependent and cannot be grasped : its degree of freedom is equal to 0 . 
Intermediary elements of degree equal to 1 can also be created : these are points on objects, like a point on a circle or on a straight line, they remain on this object when the Cabri-drawing is dragged.

This notion of freedom due to the drag mode and to the necessity of a construction process is not included in the theory of euclidean geometry. It introduces new questions such as what is the trajectory of a point on an object when the Cabri-drawing is dragged? This is answerable only through the decision of the designers of the software, but which was not completely arbitrary. The behaviour of the point on an object cannot be too remote from the expectations of the user. Because a figure in

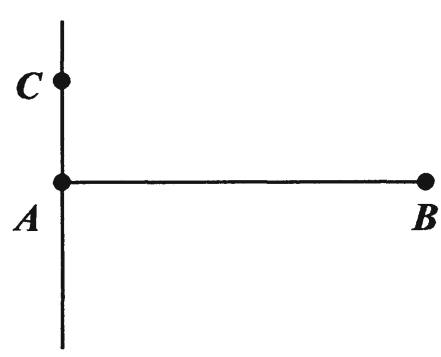

Figure 3

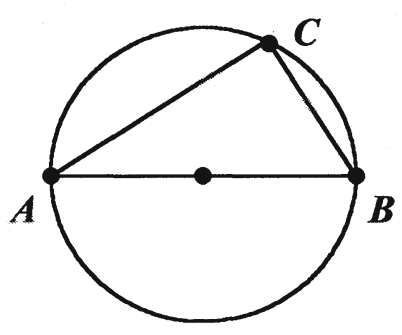

Figure 4

Euclidean geometry is invariant through a similarity, it has been decided to keep the ratio IA/IB constant for a point I on a segment AB. As a consequence, it would be non relevant to infer properties of a point on an object from observing its trajectory in the drag mode.

The same geometrical figure can give rise to different Cabri-figures. A right triangle $\mathrm{ABC}$ right in $\mathrm{A}$ can be constructed by choosing $\mathrm{A}$ and $\mathrm{B}$ as free points and $C$ on the perpendicular line to $A B$ at $A$ (fig. 3) or by choosing $\mathrm{B}$ and $\mathrm{C}$ as free points and $\mathrm{A}$ as point on the circle with diameter $\mathrm{BC}$ (fig 4).

Construction aspects and dependence relations play an important role in the way in which the Cabri-figure may be handled. By the way functional aspects of the elements of a figure are emphasized: a perpendicular bisector must be seen as depending on one variable (a segment) or two variables (two points), a perpendicular line must be seen as depending on two variables, a point and a line. 


\section{LEARNING GEOMETRY IN A CABRI-ENVIRONMENT: SOME HYPOTHESES}

We believe that the traditional teaching of geometry emphasizes the role of theoretical knowledge and ignores or underestimates the relations between drawing and geometrical theory. Geometry is not presented as knowledge allowing the interpretation of visual phenomena or even the control and prediction of them.

The role of drawings is seen more as an auxiliary illustration of geometrical concepts. Pupils are not taught how to cope with a drawing, they are not taught how to interpret a drawing in geometrical terms, or how to distinguish the spatial properties which are pertinent from a geometrical point of view from those which are only attached to the drawing. In other words they are not taught how to distinguish spatial properties which are necessary from a geometrical point of view from properties which are only contingent in the drawing. This is the reason why there may be some misunderstandings between pupils and their teacher. When the pupils are given a construction task, the teacher perceives it as a task involving the use of geometry, whereas for the pupils it may be a task of drawing. For example, when asked to draw a tangent line to a circle passing through a point $\mathrm{P}$, the pupils often rotate a straight edge around $\mathrm{P}$ so that it touches the circle. This is not based on geometry but on perception.

For the same reasons when pupils are asked to prove spatial property, it may be difficult for them to understand why they are not allowed to infer properties directly from the drawing. These behaviours of the pupils have been described by researchers in different countries such as Hillel \& Kieran [4], Schoenfield [5], Fishbein [6] and Mariotti [7].

We propose the hypothesis that by designing specific tasks in a software environment like Cabri-géomètre it is possible to promote the learning of geometrical knowledge as a tool for interpreting, explaining, producing and predicting some visual phenomena. And relating to the hypotheses stated at the beginning of this paper, the software environment plays a double role, in that it is the source of tasks and it offers ways of actions and feedback to the pupils solving the task.

\section{NEW PROBLEMS RAISED BY THE COMPUTER ENVIRONMENT}

On the one hand, the dynamic treatment of the Cabri-drawings raises new categories of problems in geometry itself, while on the other hand the 
environment through its specific possibilities allows to give problems to learners which could not be proposed in a paper and pencil environment.

\section{New kinds of problems in geometry}

Generecy of a construction. Some constructions depend on the mutual spatial position of elements of the figure which can change under the effect of the drag mode. For example, constructing the tangent lines at a circle from point $\mathrm{P}$ outside a circle is not made by the same process as when the point is on the circle. It means that when dragging the point $P$ until sticking it at the circle, the construction process does not provide the tangent line for the end-point of the trajectory of P.

Order of points. When coping only with static drawings, it is difficult to give a meaning to a simple question such as "how to determine by means of a geometrical construction what point among three points on a straight line D is between the two others when these points are moving on D?" It is easy to answer perceptively but the perception provides neither a generic way of solution nor a geometrical process.

Conditional objects. The dynamic feature of a Cabri-drawing may also raise a kind of question which until recently was asked only in a formal setting : how to produce a Cabri-drawing which only exists if the elements defining it belong to a subset of the plane.

The three categories of problems presented here emerge from the fact that the Cabri-drawing is a new kind of representation of geometrical object with specific behaviour produced and controlled by geometrical knowledge:

- it can be moved by direct manipulation (and not by means of a symbolic language) and it keeps its geometrical properties in the movement;

- it is produced by a geometrical algorithm based on geometrical primitives.

This clearly shows that new kinds of representations may give rise to new kinds of problems which call for geometrical knowledge to be solved. Indeed, tasks may be designed for pupils that are specific to the Cabri-environment and which can only be solved by using geometrical knowledge. According to our hypothesis concerning the role of problems solving this kind of task may promote learning for the pupils. What is of interest is to design tasks calling for a use of knowledge which are impossible in traditional environments. 


\section{New problems for the learners}

With regard to the relations between drawing and geometry, several kinds of tasks can be distinguished:

(i) moving from a verbal description of a geometrical figure to a drawing: this refers in a paper and pencil environment to the classical construction tasks in which pupils have to produce the drawings of geometrical objects given by a verbal description;

(ii) explaining the behaviour of drawings by means of geometry which corresponds to moving from drawing to verbal description and explanation:

a) interpreting drawings in geometrical terms; this occurs in tasks in which pupils have to prove why a spatial property giving rise to a visual evidence is verified by a drawing;

b) predicting a visual phenomenon as in problems involving a locus of points;

(iii) reproducing a drawing or transforming a drawing by using geometry.

These three kinds of tasks become different in Cabri-géomètre because of the specificities of a Cabri-drawing. Examples of such tasks are given elsewhere [8], [9], [10], [11] and [12].

Briefly speaking tasks (i) and (iii) should a priori require the use of geometrical knowledge and tasks (ii) seem to motivate the need of proof. All three kinds of tasks involve several aspects of the relations between drawings and geometrical properties

\section{PUPILS' SOLVING BEHAVIOURS WHEN INTERACTING WITH THE SOFTWARE ENVIRONMENT}

Although theoretical objects are now reified or embodied in a material environment, the learner will not immediately have access to the meaning intended by the designer of the environment or the teacher. It has often been stated about the Logo turtle that the pupils did not conceptualize the notion of angle simply because they were faced with a task including the construction of angles for instance (Hoyles \& Sutherland [13] and Hillel \& Kieran [4]. The learners construct a representation of the functioning of the software and of the tasks which are given to them which may differ from the expected representation.

Numerous observations of pupils of grade 8 and 9 working with Cabri-géomètre have given evidence about the pupils' solving processes in 
this environment, about these use of their primitives and of the drag mode. Briefly, pupils moved from purely visual to geometrical strategies when solving a problem, and the features of the software played an important role in the move from one strategy to the next. It was particularly interesting to observe strategies which mixed visual and geometric steps. At the same time, pupils used the drag mode both as a validity criterion for their constructions, as well as a means of exploration. All observations convinced us that some of the learning was particular to the nature of Cabri, and the software enabled us to observe the process or evolution, of their learning. The evidence and discussion are reported in details elsewhere [8] and [14].

\section{CONCLUSION}

Software provides rich environments for modelling or embodying the mathematical objects. The study of the example of Cabri-géomètre in the case of geometry stresses the necessity of analysing a new kind of relation to knowledge which is constructed by the pupils through a software environment. New tasks can be used by the teacher in order to promote learning. But what is their meaning for the pupils? It appears that the visual feedback based on geometry plays an important role in the development of pupils' learning, both by showing the inadequacy of strategies and as giving evidence for some visual phenomena. It also appears that the pupils could use more extensively the conjunction of drag mode and geometrical primitives in order to receive more sophisticated feedback. For this reason, we emphasize the relations between visual and geometrical phenomena through new interface possibilities. In Cabri-like software the scope of visual phenomena is dramatically enlarged and at the same time these phenomena are controlled and produced by theory. They could be used more in the teaching and learning of geometry, and could give a greater meaning to geometrical tasks set for pupils, which up to now are very often viewed by pupils as school tasks without any relation to visual phenomena.

\section{REFERENCES}

1. Brousseau, G. (1986) Fondements et méthodes de la didactique des mathématiques. Recherches en didactqiue des mathématiques 7 (2), 33-115. 
2. Pea, R. (1987) Cognitive Technologies for Mathematics Education. In Schoenfeld, A. (ed), Cognitive Science and Mathematical Education, Hillsdale, N J, LEA publishers, 89-122.

3. Parzysy, B. (1988) Knowing vs Seeing. Problems of the plane representation of space geometry figures. Education Studies in Mathematics, 19 (1), 79-92.

4. Hillel, J \& Kieran, C. (1988) Schemas used by 12 year olds in solving selected turtle geometry tasks. Recherches en didactique des mathématiques, 8 (1.2), 61-102.

5. Schonfeld, A. (1986) Students' beliefs about Geometry and their effects on the students' geometric performance. Paper presented at the tenth international Conference of the group Psychology of Mathematics Education, London

6. Fishbein, E. (1993) The theory of figural concepts. Educational studies in Mathematics, 24 (2) 139-162.

7. Mariotti, A. (1991) Age variant and invariant elements in the solution of unfolding problems. Proceedings of PME XV, Furinghetti F. (ed), II, Assisi, Italie, 389-396.

8. Laborde, C., \& Laborde, M.J. (1993) Designing tasks for learning geometry in a computer based environment: the case of Cabri-géomètre. In: Proceedings of the Technology for Mathematics Teaching Conference, Birmingham, U.K. (in press).

9. Capponi, B. (1993) Modifications des menus dans Cabri-géomètre. Des symétries comme outils de construction, Petit x, n 33, pp. 37-68

10. Guillerault, M. (1991) La gestion des menus dans Cabri-géomètre, étude d'une variable didactique. Memoire de DEA de didactique des disciplines scientifiques, Laboratoire LSD2-IMAG, Université Joseph Fourier, Grenoble

11. Bergue, D. (1992) Une utilisation du logiciel "Géomètre" en 5ème. Petit x, IREM de Grenoble, 29, 5-13

12. Boury, V. (1993) La distinction entre figure et dessin en géomètrie : etude d'une "boîte noire" sous Cabri-géomètre. Rapport de stage du DEA de sciences cognitives, Equipe DidaTech, LD2 IMAG, Université Joseph Fourier, Grenoble 
13. Hoyles, C. \& Sutherland R. (1990) Pupil collaboration and teacher intervention in the LOGO environment. Journal für Didaktik der Mathematik, 11 (4), 323-43

14. Berthelot, R \& Salin, M.H. (1992) L'enseignement de l'espace et de la géomètrie dans la scolarité obligatoire, Thèse de l'Université Bordeaux 1.

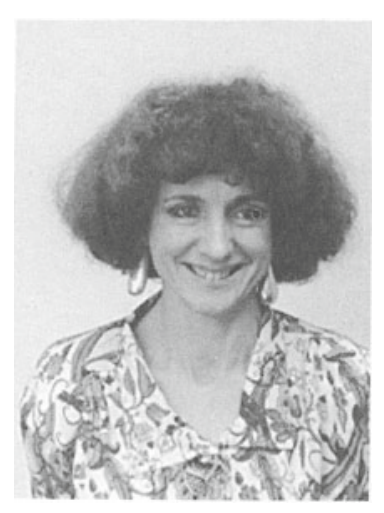

Colette Laborde graduated in mathematics from the Ecole Normale Supérieure, obtaining a "Doctorat es Sciences" in mathematics education at the Scientific University of Grenoble, specialising in language problems in mathematics teaching and learning. She is a full professor at the University Teaching Institute and a member of the mathematics education research group at the Institute for Applied Mathematics and Computer Sciences (IMAG). Colette Laborde is head of the doctoral programme of mathematics and science at the University of Grenoble and currently a full researcher of the CRNS.

Jean-Marie Laborde graduated in mathematics from the Ecole Normale Supérieure in Paris and started his research work at the Institute for Applied Mathematics and Computer Sciences (IMAG), specialising in the use of geometric methods for the study of different classes of graphs, especially hypercubes, and also automatic theorem proving. With others he started the Cabrigéomètre project in 1981, initially as an environment for graph-theory. In 1987 a number of students and young researchers joined the project to start Cabri-géomètre, which a year later was nominated as Educational Software of the Year by the Apple Company. In 1982, Jean-Marie Laborde founded the Laboratory for Discrete Mathematics and Research at Grenoble University. He has been guest professor at several universities and is currently Research Director of the CRNS and Head of the Cabri-géomètre Project at IMAG. 